\title{
Cabanyal. Los ciudadanos en la protección de los valores de su medio urbano
}

\author{
Jesús Jiménez López, letrado de la Junta de Andalucía exc.
}

Todos estamos acostumbrados a los tradicionales conflictos técnicos, jurídicos y sociales que se suscitan en torno a la protección del patrimonio histórico, con carácter general, y en particular en aquellos casos, los más, donde el objeto y alcance de la protección se asientan en los denominados conceptos jurídicos indeterminados y la revisión jurisdiccional de su concreción. Aún así, es difícil encontrar un supuesto en el que el conjunto de competencias, funciones, tutela y conceptos se hayan cuestionado tanto como el que analizamos. Hablamos de la protección del barrio del Cabanyal, en Valencia.

Efectivamente, analizada la evolución del caso desde la perspectiva que da el tiempo y, en cierto modo, la distancia, nos encontramos ante la protección de un bien, el barrio del Cabanyal, sobre el que han intervenido o actuado, como objeto específico más allá de normas generales, el Ayuntamiento de Valencia', la Administración de la comunidad autónoma de Valencia², el Parlamento de Valencia ${ }^{3}$, la Administración General del Estado ${ }^{4}$, los tribunales superiores de justicia de Madrid ${ }^{5}$ y Valencia ${ }^{6}$, el Tribunal Supremo ${ }^{7}$ y el Tribunal Constitucional. Igualmente se ha recabado la intervención de los órganos e instituciones consultivas más relevantes de España en materia de protección del patrimonio histórico ${ }^{8}$.

Toda esta actividad de revisión administrativa y jurisdiccional ha sido promovida y sostenida por el Instituto de Defensa de Intereses Patrimoniales, Culturales y Artísticos del Cabanyal-Canyamelar (INDIPCACC-Salvem el Cabanyal) y, en menor medida, por la Asociación de Vecinos PAVIMAR y la Federación de Asociaciones de Vecinos de Valencia?.

El primer problema que nos encontramos es el sistematizar todo el proceso ante la numerosa actividad desarrollada por los ciudadanos y los poderes públicos antes citados.

A modo de aproximación, podemos decir que el objeto de la protección, como patrimonio histórico, se definió inicialmente en el Decreto 57/1993, de 3 de mayo, del Gobierno valenciano, por el que se declaró bien de interés cultural el conjunto histórico de Valencia. En él se identifica el denominado barrio del Cabanyal, el cual, resultado de las transformaciones urbanisticas de final del siglo XIX, se conforma como "una peculiar trama en retícula derivada de las alineaciones de las antiguas barracas, en las que se desarrolla una arquitectura popular de clara raigambre eclecticista".
Igualmente, también a los efectos de delimitar el origen y objeto de la controversia, el Plan Especial de Protección y Reforma Interior Cabanyal-Canyamelar, aprobado por la comunidad valenciana el 2 de abril de 2001, considera como objetivo "resolver de manera adecuada la conexión con el frente marítimo de la ciudad de Valencia", teniendo a su vez como objeto la "regeneración y revitalización de los barrios del Cabanyal y Canyamelar". La ejecución del mismo determina la "...construcción de una nueva avenida de 48 metros de anchura (Av. Blasco lbañez) bordeada de bloques de nuevas edificaciones de cinco plantas (...); demolición de todas las viviendas que se encuentran en el espacio a ocupar por la avenida de 48 metros y las nuevas edificaciones que surgirán a lo largo de la avenida..." ${ }^{10}$.

La declaración, mediante Decreto 57/1993, donde se concreta el objeto de la protección, ante las controversias surgidas sobre el efecto que el PEPRI puede tener sobre el mismo, ha pretendido ser modificada, para solucionar el problema de origen.

En este sentido, la Ley 2/2010, de 31 de marzo, sobre medidas de protección y revitalización del conjunto histórico de la ciudad de Valencia, sin perjuicio del resto de contenidos que después analizaremos, establece en su disposición derogatoria única que "queda derogada cualquier norma de igual o inferior rango que se oponga a lo establecido en la presente Ley (apartado 1)". Continúa diciendo, en su apartado 2, que "de una forma especial, el Decreto 57/1993, de 3 de mayo, del Consell, por el que se declaró bien de interés cultural al conjunto histórico de Valencia, deberá entenderse modificado o derogado en cualquier aspecto que pudiera oponerse a lo establecido en la presente Ley". Y concluye que "así mismo, el Decreto 57/1993, de 3 de mayo, del Consell, por el que se declaró bien de interés cultural al conjunto histórico de Valencia, deberá entenderse modificado o derogado en cualquier aspecto que pudiera oponerse a la total ejecución del Plan Especial de Protección y de Reforma Interior del Cabanyal-Canyamelar, aprobado definitivamente por Resolución de 2 de abril de 2001, del conseller de Obras Públicas, Urbanismo y Transportes, ya que dicho Plan produce una mejora de la relación del barrio del Cabanyal con su entorno territorial o urbano y evita usos degradantes para el propio conjunto."

Como se observa, mediante esta ley, se pretende hacer del Plan Especial de Protección y Reforma Interior, no un instrumento de protección del conjunto histórico, sino un instrumento de delimi- 


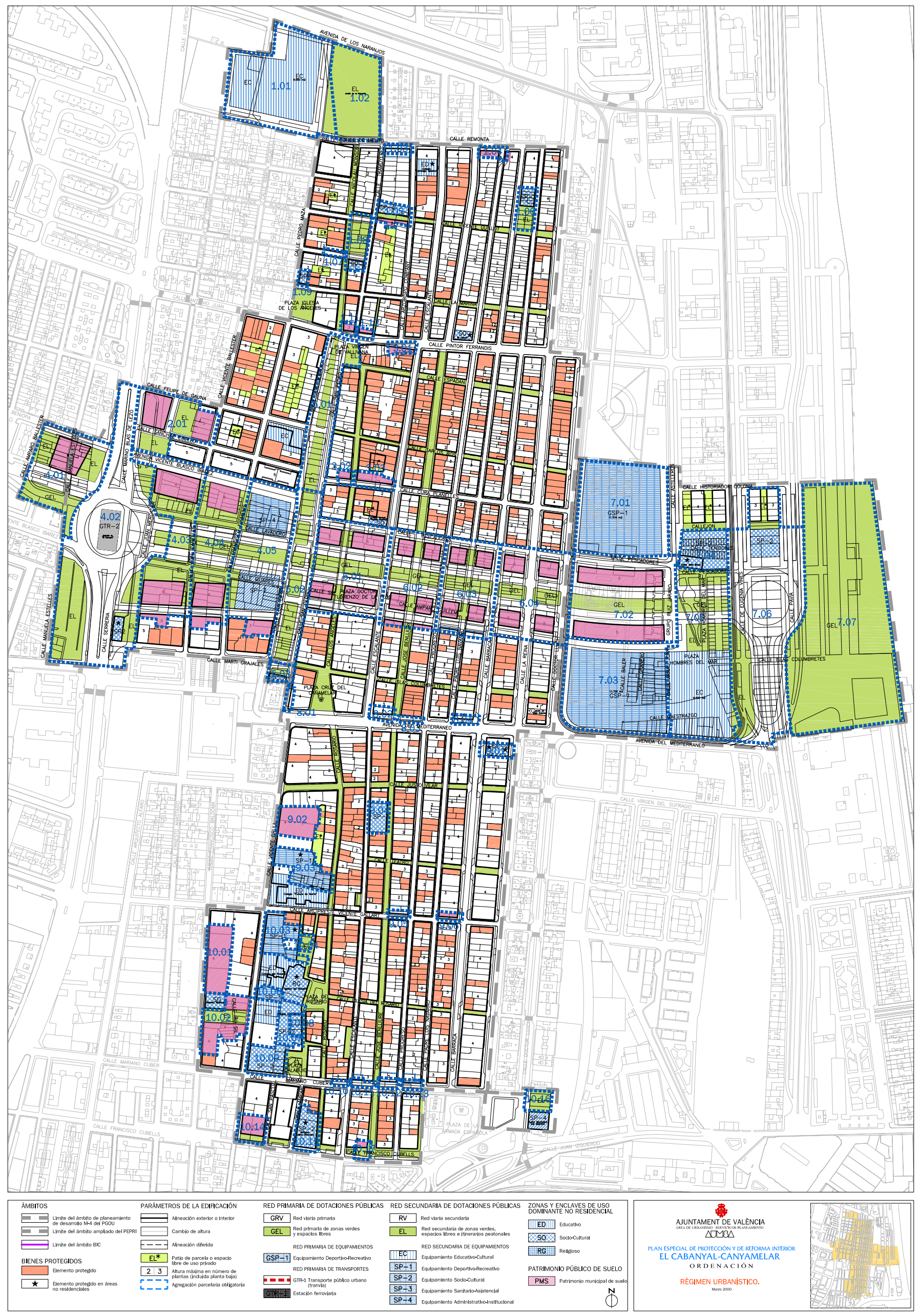

PEPRI. Régimen urbanístico. Fuente: Web oficial Plan Cabanyal-Canyamelar (hitp://www. plancabanyal.es/plano. php?:id=TXC9PQ) 
tación del objeto de la misma. Reduce espacialmente el objeto del conjunto histórico en la medida que la modificación de determinados espacios, comprendidos en la declaración originaria, está incluida en el Plan Especial.

Más allá de consideraciones de oportunidad, la ley pretende terminar con el debate planteado.

Los elementos cuya protección resulte contradictoria con el Plan Especial dejarán de estar protegidos. Por otro lado, al haberlo resuelto mediante una norma de rango legal, se sustrae la delimitación de la protección, su objeto, de la participación de los ciudadanos y las entidades que los representan, de las instituciones consultivas y del análisis jurisdiccional propio de la concreción de los conceptos jurídicos indeterminados. En cualquier caso sacrifica las posibilidades impugnatorias ante la jurisdicción ordinaria a instancias de los ciudadanos interesados.

En realidad, la ley rompe con un consenso inicial, sobre el que todas las partes implicadas estaban de acuerdo, al menos en apariencia. Efectivamente, todas las administraciones públicas y los ciudadanos se mostraban de acuerdo en la singularidad del barrio del Cabanyal, la de su estructura urbana y la tipología edificatoria, y la necesidad de su preservación. La discrepancia, en apariencia, se reducia a la forma en que se garantizaba su preservación.

Sobre la vigencia y aplicación de la norma volveremos más adelante.

Entrando en el fondo del asunto, la cuestión en inicio suscitada se asienta en la medida en que el Plan Especial de Protección y Reforma Interior de 2001 cumple la finalidad que sus redactores le atribuyen, es decir, si contribuye a la protección del barrio o supone un quebranto, un menoscabo de sus valores.

Para ello es necesario, siquiera someramente, realizar un breve examen normativo:

Establece el artículo 21 (protección y conservación de conjuntos históricos) de la Ley 16/1985, de 25 de junio, de patrimonio histórico español:

"2. Excepcionalmente, el Plan de protección de un Conjunto Histórico podrá permitir remodelaciones urbanas, pero sólo en caso de que impliquen una mejora de sus relaciones con el entorno territorial o urbano o eviten los usos degradantes para el propio Conjunto.

3. La conservación de los Conjuntos Históricos declarados Bienes de Interés Cultural comporta el mantenimiento de la estructura urbana y arquitectónica, así como de las caracteristicas generales de su ambiente. Se considerarán excepcionales las sustituciones de inmuebles, aunque sean parciales, y sólo podrán realizarse en la medida en que contribuyan a la conservación general del carácter del Conjunto. En todo caso, se mantendrán las alineaciones urbanas existentes".
Por su parte, el artículo 39 (planes especiales de protección) de la Ley 4/1998, de 11 de junio, de patrimonio cultural valenciano, establece:

"2. Los Planes Especiales de Protección de los Conjuntos Históricos y sus modificaciones, tendrán en cuenta los siguientes criterios:

a) Se mantendrá la estructura urbana y arquitectónica del conjunto y las características generales del ambiente y de la silueta paisajistica.

No se permitirán modificaciones de alineaciones, alteraciones de la edificabilidad, parcelaciones ni agregaciones de inmuebles, salvo que contribuyan a la mejor conservación general del conjunto.

b) No obstante lo dispuesto en el apartado anterior, con carácter excepcional, el Consell podrá autorizar, oídos al menos dos de los organismos a que se refiere el artículo 7 de esta Ley, que los Planes Especiales de Protección de los Conjuntos históricos prevean modificaciones de la estructura urbana y arquitectónica en el caso de que se produzca una mejora de su relación con el entorno territorial o urbano o se eviten los usos degradantes para el propio conjunto o se trate de actuaciones de interés general para el municipio o de proyectos singulares relevantes" (el contenido de esta letra $b$ fue introducido mediante la Ley 5/2007, de 9 de febrero).

Como manifiesta el mismo documento, el Plan Especial de Protección y Reforma Interior de 2001 considera como objetivo "resolver de manera adecuada la conexión con el frente maritimo de la ciudad de Valencia", teniendo a su vez como objeto la "regeneración y revitalización de los barrios del Cabanyal y Canyamelar".

El modo que propone es la "...construcción de una nueva avenida de 48 metros de anchura (Av. Blasco Ibáñez) bordeada de bloques de nuevas edificaciones de cinco plantas (...); demolición de todas las viviendas que se encuentran en el espacio a ocupar por la avenida de 48 metros y las nuevas edificaciones que surgirán a lo largo de la avenida..."

Como consecuencia, las asociaciones vecinales precitadas iniciaron dos líneas de actuación fundamentales: recabar una revisión jurisdiccional del Plan Especial de Protección y Reforma Interior, mediante la impugnación del acuerdo de su aprobación definitiva; e instar una revisión por la Administración General del Estado, en el ejercicio de sus competencias en materia de expoliación.

La primera iniciativa de los colectivos ciudadanos y científicos interesados se concretó en la impugnación de la resolución de 2 de abril de de 2001, por la que se aprobó definitivamente el Plan Especial de Protección y Reforma Especial del Cabanyal-Canyamelar, así como el informe de 5 de enero de 2001 que le sirve de soporte.

Efectivamente, interpuesto recurso contencioso administrativo, se dictó una primera sentencia por el Tribunal Superior de Justicia de Valencia (recurso 984/2001) de 1 de octubre de 2004. La sentencia 
es desestimatoria del recurso y se asienta en que la propuesta no contradice las previsiones del artículo 39.2.a) de la Ley de patrimonio cultural valenciano, considerando que, mediante las alteraciones propuestas y conforme a las consideraciones de los informes técnicos incorporados al expediente, se contribuye a la mejor conservación del conjunto" ${ }^{11}$ excepción prevista en el citado precepto.

Esta sentencia fue objeto de diversos recursos de casación interpuestos por la asociación Instituto de Defensa de Intereses Patrimoniales, Culturales y Artísticos del Cabanyal Canyamelar, la Asociación de Vecinos PAVIMAR y la Federación de Asociaciones Vecinos de Valencia, resueltos mediante las sentencias del Tribunal Supremo de 12 y 13 de marzo, y de 16 de diciembre de 2008, respectivamente.

Los recursos fueron desestimados, si bien el análisis jurídico de fondo se vio bastante limitado. Considerando el Tribunal Supremo cuestión central del debate en el proceso de instancia el "determinar si el Plan Especial impugnado se acomoda o no las determinaciones del Artículo 39.2 de la Ley 4/1998 del Patrimonio Cultural Valenciano" (fundamento jurídico séptimo), queda fuera de su análisis por referirse a un precepto del ordenamiento valenciano, excluido por tanto de casación ${ }^{12}$.

Por otro lado y además, en relación con el motivo impugnatorio referido a la vulneración de las normas relativas a la interdicción de la expoliación, el Tribunal Supremo, después de analizar las competencias estatales ${ }^{13}$, el concepto y alcance de expoliación ${ }^{14}$, concluye: la actuación de las administraciones competentes -Ayuntamiento y comunidad autónoma de Valencia- no se debe de considerar irracional o arbitraria; y "no hay constancia de que haya habido denuncia ni procedimiento administrativo sobre una posible expoliación ni, por tanto, un pronunciamiento de la Administración Estatal acerca de tal cuestión".

La segunda línea de actuación tiene que ver precisamente con la responsabilidad y competencia del Estado en relación con la protección del patrimonio histórico para evitar su expoliación, de acuerdo con lo establecido en el artículo 149.1.28 de la Constitución Española, desarrollado en este punto por el artículo 6.b) de la Ley 16/1985, de 25 de junio, de patrimonio histórico español.

Aún pudiendo ser cierto que el Tribunal Supremo no tenía constancia de acción administrativa alguna de los interesados, para recabar la intervención del Estado por considerar que el PEPRI, su ejecución, puede considerarse expoliación, la realidad es que la actividad de las entidades ciudadanas interesadas habia sido también intensa en esta dirección.

Efectivamente, desde el 27 de septiembre de 1999, antes por tanto incluso de la aprobación provisional del PEPRI, habian reclamado la intervención del Estado en ejercicio de estas competencias. La denuncia fue reiterada en diversas ocasiones acompañando informes técnicos en los que fundamentaban la petición de intervención del Estado.
Este procedimiento inicial terminó mediante escrito de 29 de enero de 2001, de la Subdirección General de Protección del Patrimonio Histórico, del Ministerio de Cultura, en el que comunicaba a los denunciantes que "la Generalidad Valenciana ha exigido aclaraciones al Ayuntamiento y luego ha acordado, en base a su autonomía y competencia exclusiva, informar favorablemente el proyecto de Plan Especial de protección y Reforma Interior del Cabanyal Canyamelar aprobado por el Ayuntamiento, sin que vulnere lo prescrito en la Ley de Patrimonio Valenciano y sin que exista tutela superior del Estado."

La escueta resolución fue objeto de recurso contencioso administrativo por la asociación INDIPCACC, resuelto por la sentencia de 27 de septiembre de 2004 (recurso 799/2001) de la Sala de Contencioso Administrativo del Tribunal Superior de Justicia de Madrid ${ }^{15}$. La sentencia anula la resolución impugnada obligando a retrotraer el procedimiento al objeto de que, previa la tramitación que proceda, se pronuncie sobre el fondo del asunto, sobre la concurrencia o no de expoliación, de acuerdo con las atribuciones que sobre la misma tiene el Estado. Esta decisión fue confirmada a su vez por el Tribunal Supremo en su sentencia de 25 de mayo de 2009 (recurso 257/2001).

En ejecución de la sentencia, el Ministerio de Cultura dicta la orden CUL/3631/2009, de 29 de diciembre, por la que se resuelve el procedimiento de expoliación del conjunto histórico del Cabanyal, tras una intensa actividad de asistencia de los principales órganos consultivos del Estado en materia cultural.

La citada orden declara que el Plan Especial de Protección y Reforma Interior de Cabanyal-Canyamelar determina la expoliación del conjunto histórico del Cabanyal. En consecuencia ordena al Ayuntamiento de Valencia la suspensión de la actuación y a la comunidad autónoma vigilar su cumplimiento por la corporación municipal. Todo ello hasta que el PEPRI se adapte para garantizar los valores que motivaron la calificación del Cabanyal como conjunto histórico.

Es conveniente la completa reproducción del apartado quinto, motivación de la orden de protección y preservación:

"Quinto. -Examinadas las consideraciones anteriores, y en base a los informes referenciados, debe concluirse que el PEPRI constituye un expolio del conjunto histórico del Cabanyal por los siguientes motivos:

1. El PEPRI ha sido dictado prescindiendo de toda consideración por los valores histórico-artísticos que motivaron la protección del conjunto histórico del Cabanyal, lo cual informa todo su contenido. 2. Consecuencia de lo anterior, las determinaciones del PEPRI suponen una alteración del conjunto histórico del Cabanyal que desfiguran el mismo hasta el punto de hacer perder a éste su propio carácter -«su peculiar trama de retícula»- en beneficio de una determinada opción de trazado urbanístico (articulación de un nuevo barrio en torno a una gran avenida que conecte el resto de Valencia con el mar) incompatible con la protección del conjunto histórico. 
3. Que dicha opción urbanistica, no sólo es incompatible con el mantenimiento de los valores del conjunto histórico, sino que ha sido adoptada apriorísticamente por el Ayuntamiento obviando los principios de proporcionalidad y mínima intervención, sin el debido estudio de las distintas soluciones técnicas posibles, y sin motivar adecuadamente la elección de la solución definitiva de entre las existentes. 4. Que las determinaciones del PEPRI consistentes en modificación de alineaciones, alteraciones de la edificabilidad y demás actuaciones vedadas, como regla general, por la normativa de protección de patrimonio histórico, no contribuyen a la mejor conservación general del conjunto, entendido éste como el definido por los valores del conjunto histórico, sino que únicamente están al servicio de la consecución de una determinada solución de política urbanistica. 5. Y que, adicionalmente a las anteriores consideraciones, las determinaciones del PEPRI comportan una serie de derribos singulares de bienes de gran valor histórico-artístico que contribuyen al deterioro del conjunto y agravan la desfiguración de aquellos que motivaron la protección del conjunto histórico."

La orden del Ministerio de Cultura ${ }^{16}$ ha sido recurrida ante la Audiencia Nacional, autos 92/2010, órgano jurisdiccional que desestimó la petición de suspensión cautelar mediante autos de 9 de abril y 24 de mayo de 2010 (súplica), siendo confirmados por la sentencia del Tribunal Supremo de 28 de junio de 2011. No consta sentencia sobre el fondo del asunto a la fecha de estas líneas.

Además de esta actividad impugnatoria, el Gobierno valenciano aprueba el Decreto Ley 1/2010, de 7 de enero, de medidas de protección y rehabilitación del conjunto histórico de la ciudad de Valencia, derogada por la Ley 2/2010, de 31 de marzo, con el mismo título y alcance.

La intención de la ley resulta directamente de su contenido en contraste con las declaraciones de la orden del Ministerio de Cultura. Asi establece:

\section{"Artículo 1 (Objeto)}

1. Se declara expresamente que el Plan Especial de Protección y de Reforma Interior del Cabanyal-Canyamelar (...) no perjudica, daña, deteriora o menoscaba de cualquier otro modo la protección del conjunto histórico de la ciudad de Valencia acordada mediante el Decreto 57/1993, de 3 de mayo, del Consell, que declaró a dicho conjunto histórico bien de interés cultural, de forma que el citado Plan no pone en peligro de pérdida o destrucción ninguno de los valores del conjunto histórico de la ciudad de Valencia ni perturba el cumplimiento de su función social sino que, antes bien, revitaliza la ciudad de Valencia en uno de sus barrios históricos más importantes y mejora la calidad de vida del mismo y el disfrute de los valores históricos del barrio del Cabanyal por sus vecinos y por el resto de ciudadanos de Valencia y de la Comunitat Valenciana.

2. Se reconoce expresamente la plena compatibilidad entre la total ejecución del Plan Especial de Protección y de Reforma Interior del Cabanyal-Canyamelar (...) y las razones que dieron lugar a la inclu- sión del núcleo original del ensanche del Cabanyal dentro de las áreas afectadas por la declaración de bien de interés cultural del conjunto histórico de Valencia, de forma que la total ejecución del citado Plan no pone en peligro de pérdida o destrucción ninguno de los valores del conjunto histórico de la ciudad de Valencia ni perturba el cumplimiento de su función social sino que, antes bien, revitaliza la ciudad de Valencia en uno de sus barrios históricos más importantes y mejora la calidad de vida del mismo y el disfrute de los valores históricos del barrio del Cabanyal por sus vecinos y por el resto de ciudadanos de Valencia y de la Comunitat Valenciana.

\section{Artículo 2 (Autorización)}

Se autorizan expresamente las actuaciones e intervenciones sobre la estructura urbana y arquitectónica del conjunto histórico de Valencia que prevé el Plan (...), ya que dicho plan produce una mejora de la relación del barrio del Cabanyal con su entorno territorial o urbano y evita usos degradantes para el propio conjunto.

\section{Artículo 3 (Ejecución del plan especial)}

Los órganos y personal al servicio de las administraciones públicas competentes para la ejecución del Plan (...) realizarán todos los actos que sean necesarios para la total ejecución del citado Plan conforme a lo previsto en el mismo y en la presente Ley, sin que tales actos de ejecución puedan verse menoscabados de cualquier modo por los actos o acuerdos de otras administraciones públicas".

Además, como se indicaba al principio, es relevante su efecto derogatorio sobre el Decreto 57/1993, de 3 de mayo, de declaración del conjunto histórico de Valencia, así como la modificación que se impone del mismo en lo que se oponga al Plan Especial de Protección y Reforma Interior del Cabanyal-Canyamelar (disposición derogatoria única, apartado 2).

Por si quedaba alguna duda, se retrotraen los efectos de la ley, en lo que pudiera ser necesario, al 11 de mayo de 1993, fecha de la entrada en vigor del precitado Decreto 57/1993, y al 2 de abril de 2001, fecha de aprobación definitiva del PEPRI (disposición adicional segunda, párrafo segundo).

Frente a la Ley del Parlamento de Valencia, la Administración General del Estado ha interpuesto recursos de inconstitucionalidad números 803/2010 (Decreto Ley) y 2977/2010, contra los artículos 1, 2 y 3, disposición derogatoria, apartado 2, y disposición adicional segunda, párrafo segundo. La interposición al amparo del artículo 161.2 de la Constitución provocó la suspensión de los preceptos impugnados.

No habiéndose dictado sentencia aún, es relevante el análisis de la cuestión desde el punto de vista de las medidas cautelares, en la medida en la que el Tribunal Constitucional se ha pronunciado sobre la procedencia de mantener la suspensión de los preceptos impugnados.

Así, el Tribunal Constitucional acordó, mediante auto de 28 de julio de 2010 (auto 104/2010), mantener la suspensión excepcional. 


\section{La decisión se sostiene en los siguientes argumentos:}

- Las decisiones contenidas en los preceptos de la Ley 2/2010 pueden obstaculizar, más allá de la controversia trabada en cuanto al fondo del asunto, el desenvolvimiento de las competencias estatales ejercidas al dictarse la orden CUL/3631/2009, de 29 de diciembre, impidiendo la plena efectividad de la decisión estatal en relación con la eventual expoliación que sufriría el conjunto histórico del Cabanyal. Por tanto, aprecia la existencia en este caso de un perjuicio actual y directo al interés general que resultaria del bloqueo de las competencias estatales en materia de expoliación.

- La ejecución del PEPRI supondría un perjuicio irreparable a los valores históricos y culturales que determinaron la declaración del conjunto histórico. Los perjuicios vinculados a la no inmediata ejecución del PEPRI las considera el Tribunal Constitucional, desde las perspectiva cautelar, susceptible de reparación.

Como se puede deducir de lo escrito hasta ahora, la cuestión más relevante, no la única, sobre la que se soportará el futuro del Cabanyal, será conocer, a la luz del Tribunal Constitucional, el alcance de la competencia del Estado en relación con la protección de los valores culturales del barrio frente a su expoliación.

Estos valores, como hipótesis considerada, estarian amenazados no sólo por las previsiones de la ejecución del PEPRI, sino por la misma modificación de la declaración contenida en el Decreto 57/1993, realizada por la Ley 2/2010, de 31 de marzo.

Siendo cierto que, con carácter general, no puede cuestionarse la competencia de las comunidades autónomas para la definición y concreción de los bienes y valores históricos, culturales y artísticos acreedores de la mayor protección de nuestro ordenamiento juridico y de los poderes públicos, también lo es que en el presente caso concurren circunstancias que revelan una acción normativa dirigida a contradecir la previa decisión adoptada.

Se ha protegido, o pretendido proteger, la decisión cuestionada mediante norma de rango legal, para debilitar la protección de unos valores que, paradójicamente, no se cuestionan.

El debate se centrará, también, en la competencia del Estado para la protección de los bienes del patrimonio histórico al objeto de evitar su expoliación. Irá referido a la habilitación del Estado para desplegar su acción protectora respecto de bienes que, teniendo valores que los enmarcan en el ámbito del patrimonio histórico, han sido despojados de su categorización como bienes más relevantes del mismo por la administración autonómica.

\section{Notas}

1 Aprobación Provisional del Plan Especial de Protección y Reforma Interior Cabanyal-Canyamelar (PEPRI) el 31 de marzo de 2001.

2 Decreto 57/1993, de 3 de mayo, del Gobierno valenciano, por el que se declara bien de interés cultural el conjunto histórico de Valencia; aprobación del PEPRI el
2 de abril de 2001; Decreto-Ley 1/2010, de 7 de enero, de medidas de protección y rehabilitación del conjunto histórico de Valencia.

${ }^{3}$ Ley 2/2010, de 31 de marzo, sobre medidas de protección y revitalización del conjunto histórico de la ciudad de Valencia.

${ }^{4}$ Resolución de la Dirección General de Bellas Artes y Bienes Culturales de 29 de enero de 2001; Orden CUL/3631/2009, de 29 de diciembre, por la que se resuelve el procedimiento de expoliación del conjunto histórico del Cabanyal.

${ }^{5}$ Sentencia del Tribunal Superior de Justicia de Madrid de 27 de septiembre de 2001 (recurso 799/2001), con relación a la resolución de la Dirección General de Bellas Artes y Bienes Culturales.

${ }^{6}$ Sentencia del Tribunal Superior de Justicia de Valencia de 1 de octubre de 2004 (recurso 984/2001) con relación a la resolución de aprobación del PEPRI, entre otras. ${ }^{7}$ Sentencia de 12 de abril de 2004, suspensión cautelar del PEPRI; sentencias de 12 y 13 de marzo de 2008, confirmando la aprobación del PEPRI; sentencia de 25 de mayo de 2009, sobre la necesidad de pronunciamiento de la Administración General del Estado sobre expoliación.

${ }^{8}$ Órganos de la administración del Ayuntamiento de Valencia, de la comunidad autónoma de Valencia, de la Administración General del Estado; Real Academia de Bellas Artes de San Fernando; Colegio de Arquitectos de la Comunidad Valenciana; Universidad Politécnica de Valencia; Consejo Internacional de Monumentos y Sitios (ICOMOS); Hispania Nostra; Consejo Superior de Colegios de Arquitectos de España; Real Academia de Historia; museo nacional de Cerámica y Artes Suntuarias González Marti de Valencia; y la Abogacia del Estado, entre otros.

${ }^{9}$ Entre otras actuaciones, participar en la tramitación del PEPRI, manifestando la oposición a su contenido; instar la participación de la Administración General del Estado ante la existencia de expoliación; elaborar recursos jurisdiccionales en todas las instancias.

${ }^{10}$ Son objetivos del plan, de acuerdo con el mismo documento PEPRI, la regeneración y revitalización de estos barrios marítimos; la reivindicación histórica de conectar Blasco lbáñez con el mar.

11 "La cuestión que ha de resolver la Sala no es la de fiscalizar la oportunidad de la medida tomada, ni sustituir el criterio de la Administración por el del demandante, igualmente legítimo, sin que éste haya acreditado la irrazonabilidad del primero, ni desde luego por el suyo propio. Siendo compatible la solución con la excepción que prevé la Ley 4/1998, única cuestión a decidir, y no si ha de prevaler la norma de Patrimonio Histórico sobre la urbanística, ya que de lo que se trata es de analizar e interpretar la norma legal autonómica citada, ha de desestimarse el presente recurso contencioso-administrativo".

${ }^{12}$ Añade además: "no se trata de que entre dos soluciones técnicas y urbanísticas enfrentadas la Sala de instancia escoja aquella que considere preferible o más acertada; lo que el órgano jurisdiccional debe dilucidar es si la decisión adoptada por la Administración cuenta con el necesario respaldo técnico y es respetuosa, en definitiva, con las normas procedimentales y sustantivas que son de aplicación" (fundamento jurídico séptimo, sentencia de 12 de marzo de 2008).

13 "El Estado ostenta, pues, la competencia exclusiva en la defensa de dicho patrimonio contra la exportación y la expoliación, y las Comunidades Autónomas recurrentes en lo restante, según sus respectivos Estatutos; sin que ello implique que la eventual afectación de intereses generales o la concurrencia de otros títulos competenciales del Estado en materia determinada no deban también tenerse presentes como limites que habrá que ponderar en cada caso concreto. (Así los títulos que resultan, v. gr. de los números 6 y 8 del artículo 149.1.)..." (fundamento juridico noveno, reproducción de la sentencia del Tribunal Constitucional 17/1991, de 31 de enero).

14 "En consecuencia -señala la STC 17/1991- la utilización del concepto de defensa contra la expoliación ha de entenderse como definitoria de un plus de protección respecto de unos bienes dotados de características especiales. Por ello mismo abarca un conjunto de medidas de defensa que a más de referirse a su deterioro o destrucción tratan de extenderse a la privación arbitraria o irracional del cumplimiento normal de aquello que constituye el propio bien según su naturaleza..."

${ }^{15}$ La Sala de lo Contencioso Administrativo del Tribunal Superior de Justicia de Madrid declara la completa ejecución de la sentencia mediante auto de 29 de diciembre de 2011.

${ }^{16}$ Debe recordarse que mediante auto de la Sala de lo Penal del Tribunal Supremo de 14 de septiembre de 2011, se ha inadmitido a trámite la querella presentada por la comunidad autónoma y el Ayuntamiento de Valencia contra la titular del Ministerio de Cultura en el momento de la firma de la orden. 